\title{
Sialic acid utilization by Cronobacter sakazakii
}

\author{
Susan Joseph, Sumyya Hariri, Naqash Masood and Stephen Forsythe
}

\begin{abstract}
Background: The Cronobacter genus is composed of seven species, and can cause infections in all age groups. Of particular concern is C. sakazakii, as this species is strongly associated with severe and often fatal cases of necrotizing enterocolitis and meningitis in neonates and infants. Whole genome sequencing has revealed that the nanAKT gene cluster required for the utilisation of exogenous sialic acid is unique to the $C$. sakazakii species (ESA_03609-13).

Sialic acid is found in breast milk, infant formula, intestinal mucin, and gangliosides in the brain, hence its metabolism by C. sakazakii is of particular interest. Therefore its metabolism could be an important virulence factor. To date, no laboratory studies demonstrating the growth of C. sakazakii on sialic acid have been published nor have there been reports of sialidase activity. The phylogenetic analysis of the nan genes is of interest to determine whether the genes have been acquired by horizontal gene transfer.
\end{abstract}

Results: Phylogenetic analysis of 19 Cronobacter strains from 7 recognised species revealed the nanAKTR genes formed a unique cluster, separate from other Enterobacteriaceae such as E. coli K1 and Citrobacter koseri, which are also associated with neonatal meningitis. The gene organisation was similar to Edwardsiella tarda in that nanE gene (N-acetylmannosamine-6-phosphate-2epimerase) was not located within the nanATK cluster. Laboratory studies confirmed that only C. sakazakii, and not the other six Cronobacter species, was able to use sialic acid as a carbon source for growth. Although the ganglioside GM1 was also used as carbon source, no candidate sialidase genes were found in the genome, instead the substrate degradation is probably due to $\beta$-galactosidase activity.

Conclusions: Given the relatively recent evolution of both C. sakazakii (15-23 million years ago) and sialic acid synthesis in vertebrates, sialic acid utilization may be an example of co-evolution by one species of the Cronobacter genus with the mammalian host. This has possibly resulted in additional virulence factors contributing to severe life-threatening infections in neonates due to the utilization of sialic acid from breast milk, infant formula, milk (oligosaccharides), mucins lining the intestinal wall, and even gangliosides in the brain after passing through the blood-brain barrier.

Keywords: Cronobacter sakazakii, Sialic acid utilisation, Sialidase, Virulence factor

\section{Background}

The Cronobacter genus is an emergent group of bacterial pathogens in the Enterobacteriaceae family. The majority of infections (bacteraemia, and urinary tract infections) are in the adult population, however the most publicized cases are severe, and frequently fatal infections in neonates and infants [1,2]. In these highly vulnerable populations, the organism is associated with necrotizing enterocolitis and a highly destructive form of meningitis in which the bacterium crosses the bloodbrain barrier and causes abscess formation in the brain

\footnotetext{
* Correspondence: stephen.forsythe@ntu.ac.uk

Pathogen Research Centre, School of Science and Technology, Nottingham Trent University, Clifton Lane, Nottingham NG11 8NS, UK
}

cavity [3,4]. The genus is composed of seven species, and multilocus sequence typing has been used to describe the diversity of the genus $[5,6]$. Evolutionary analysis suggests that the $C$. sakazakii species separated from the rest of the Cronobacter genus 15-23 million years ago (MYA) [6]. Recent whole genome studies have revealed that C. sakazakii is the only Cronobacter species that has the nanAKT gene cluster encoding for sialic acid utilization $[7,8]$. Since sialic acid is found in breast milk, infant formula, mucin lining the intestinal tract and gangliosides in the brain [9], it is plausible that this metabolism may account for the predominance of C. sakazakii in neonatal and infant infections. However

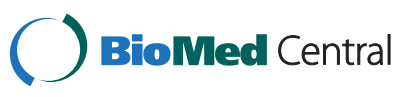


no laboratory studies investigating this trait have been undertaken to date.

Sialic acid can exist in nearly 50 different forms with the most studied being 2-keto-3-deoxy-5-acetamido-Dglycero-D-galacto-nonulosonic acid, often abbreviated as Neu5Ac. This sialic acid is generally found bound to sugars to form polysaccharides, and also can be bound to lipids or proteins to form sialo-glycoconjugates. With few exceptions, sialic acid conjugates are notably absent from many eukaryotic lineages, including most protostomes, plants, fungi, and protists. It is postulated that sialic acid synthesis evolved in animals and later emerged in bacterial pathogens and commensals either by convergent evolution or horizontal gene transfer. A number of microbial strategies have evolved which target host sialic acids for adherence, mimicry, and degradation $[10,11]$. Some bacteria can produce sialidase (or neuraminidase), encoded by $n a n H$, to cleave sialic acid from the glycoconjugate forms. This gene has low homology $(<30 \%)$ across bacterial groups, and has not been described in many organisms [12,13]. Although neonatal meningitic $E$. coli $\mathrm{K} 1$ is able to grow on sialic acid, it lacks the enzyme sialidase. However it is possibly able to obtain sufficient sialic acid from the activities of other sialidase-producing bacteria in the environment or from the host cells expressing the enzyme in conditions of inflammation [10,13].

The uptake of sialic acid through the outer cell membrane of Gram negative bacteria is by an outer membrane porin, NanC. There are three different types of transporters for the inner membrane: NanT, a major facilitator superfamily (MFS) protein; TRAP, a tripartite ATP-independent periplasmic transport system; and an ATP-binding cassette (ABC) transporter. All members of the Enterobacteriaceae studied to date have shown the presence of the single-component NanT transport system $[10,11,14]$. Once transported into the cell, the Neu5Ac lyase (NanA) converts sialic acid (Neu5Ac) into $\mathrm{N}$-acetylmannosamine (ManNAc) and phosphoenolpyruvate (PEP). NanK is an ATP-dependent kinase specific for ManNAc generating N-acetylmannosamine-6-phosphate (ManNAc-6-P). ManNAc-6-P epimerase (NanE) then converts the ManNAc-6-P into N-acetylglucosa mine-6-phosphate (GlcNAc-6-P). GlcNAc-6-P deacety lase (NagA) and glucosamine-6-P deaminase (NagB) con vert GlcNAc-6-P into fructose-6-phosphate, which is a substrate in the glycolytic pathway. NanR is the repressor that regulates the activity of these genes. The genes for the first three enzymes (nanA, nanK and nanE) are usually found clustered together forming the nan gene cluster [11]. However, there have been a few exceptions such as Citrobacter freundii and Edwardsiella tarda where the nanE gene is located in a separate region from the rest of the operon [14]. The genes encoding NagA and NagB are located adjacent to each other, but most often not necessarily in the vicinity of the nan gene cluster on the bacterial genomes [12-14]. The genes within the cluster show independent evolutionary histories. Several horizontal gene transfer events are noted in the phylogenetic trees for all three proteins. Most significantly, the NanA protein shows several possible horizontal gene transfer events between the Eukaryotes and Prokaryotes. Two examples are the clustering of Trichomonas vaginalis NanA protein sequences with Pasteu rellaceae, and the Bacteroides, Yersinia and Vibrio branching closely with the Eukarya kingdom [11,14].

The uptake of sialic acid into bacterial cells has been associated with a number of virulence factors. The bacterial glycolipid capsule is an example of molecular mimicry of the host as it aids the organism to overcome the immune responses of the host. Neonatal meningitic E. coli $\mathrm{K} 1$ uses sialic acid to decorate the cell surface, and Cronobacter does produce capsular material, especially when grown on milk [15]. Sun et al. [16] reported the O-PS gene for C. turicensis G3882 included Nacetylneuraminic acid synthetase and CMP-N-acetylneu raminic acid synthetase. However the accurate identification of this strain is uncertain as the $16 \mathrm{~S}$ rRNA gene sequence (Accession no. HQ880409) does not match other strains of $C$. turicensis.

As previously reported for the genome sequenced strain C. sakazakii BAA-894, the gene cluster encoding for a putative sugar isomerase $(\mathrm{YhcH})$ and the nanKTAR genes (encoding $\mathrm{N}$-acetylneuraminate and $\mathrm{N}$-acetylman nosamine degradation) are located at ESA_03609-13 $[7,8]$. However, the remaining nan genes for sialic acid metabolism have not been described in detail, and these previous bioinformatic studies did not identify any candidate sialidase (NanH) genes [8].

C. sakazakii is associated with infections of low birth weight neonates, and this could be linked to a number of opportunities for the organism to utilize sialic acid for growth. Human milk contains sialic acid in the form of sialyloligosaccharides which are highest in colostrum and decreases by nearly $80 \%$ over the following 3 months after birth $[9,17]$. Human milk from mothers of preterm infants contains $13-23 \%$ more sialic acid than milk from mothers of full-term infants at 3 of the 4 lactation stages. Similarly infant formulas contain sialic acid which may be bound to glycoproteins. Although the nutritional significance of sialic acid is unknown, it is plausible that it contributes to sialic acid accumulation in the brain. Breast milk also contains a variety of sialoglycans; secretory IgA, lactoferrin, and oligosaccharides. Human milk oligosaccharides are poorly digested and are substrates for intestinal bacterial metabolism, promoting bacterial growth in the intestinal tract. As a site of bacterial attachment, the intestinal microvilli of neonates 
Table 1 Distribution of the sialic acid utilisation and other related genes across the sequenced genomes of the Cronobacter genus

\begin{tabular}{|c|c|c|c|c|c|c|c|c|c|c|c|c|c|c|c|c|}
\hline Gene & Loci & Function & $\begin{array}{c}\text { C. } \\
\text { sakazakii } \\
\text { BAA-894 }\end{array}$ & $\begin{array}{c}\text { C. } \\
\text { sakazakii } \\
680\end{array}$ & $\begin{array}{c}\text { C. } \\
\text { sakazakii } \\
701\end{array}$ & $\begin{array}{c}\text { C. } \\
\text { sakazakii } \\
\text { E899 }\end{array}$ & $\begin{array}{c}\text { C. } \\
\text { sakazakii } \\
696\end{array}$ & $\begin{array}{c}\text { C. } \\
\text { malonaticus } \\
507\end{array}$ & $\begin{array}{c}C . \\
\text { malonaticus } \\
681\end{array}$ & $\begin{array}{c}\text { C. } \\
\text { turicensis } \\
564\end{array}$ & $\begin{array}{c}\text { C. } \\
\text { turicensis } \\
z 3032\end{array}$ & $\begin{array}{c}\text { C. } \\
\text { universalis } \\
581\end{array}$ & $\begin{array}{c}C . \\
\text { muytjensii } \\
530\end{array}$ & $\begin{array}{c}\text { C. } \\
\text { dublinensis } \\
582\end{array}$ & $\begin{array}{c}\text { C. } \\
\text { dublinensis } \\
1210\end{array}$ & $\begin{array}{c}\text { C. } \\
\text { condimenti } \\
1330\end{array}$ \\
\hline yhcH & ESA_03609 & $\begin{array}{l}\text { Putative sugar } \\
\text { isomerase }\end{array}$ & + & + & + & + & + & - & - & - & - & - & - & - & - & - \\
\hline nank & ESA_03610 & $\begin{array}{l}\mathrm{N} \text {-acetylmannosamine } \\
\text { kinase }\end{array}$ & + & + & + & + & + & - & - & - & - & - & - & - & - & - \\
\hline nant & ESA_03611 & $\begin{array}{l}\text { Sialic acid transporter } \\
\text { (permease) }\end{array}$ & + & + & + & + & + & - & - & - & - & - & - & - & - & - \\
\hline nanA & ESA_03612 & $\begin{array}{l}\mathrm{N} \text {-acetylneuraminate } \\
\text { lyase }\end{array}$ & + & + & + & + & + & - & - & - & - & - & - & - & - & - \\
\hline $\operatorname{nan} R$ & ESA_03613 & $\begin{array}{l}\text { Transcriptional } \\
\text { regulator }\end{array}$ & + & + & + & + & + & - & - & - & - & - & - & - & - & - \\
\hline nanc & ESA_03302 & $\begin{array}{l}\mathrm{N} \text {-acetylneuraminic } \\
\text { acid outer membrane } \\
\text { channel protein }\end{array}$ & + & + & + & + & + & - & - & - & - & - & - & - & - & - \\
\hline nanE & ESA_00529 & $\begin{array}{l}\mathrm{N} \text {-acetylmannosamine- } \\
\text { 6-phosphate 2- } \\
\text { epimerase }\end{array}$ & + & + & + & + & + & + & + & + & + & + & + & + & + & + \\
\hline nagA & ESA_02662 & $\begin{array}{l}\mathrm{N} \text {-acetylglucosamine-6- } \\
\text { phosphate deacetylase }\end{array}$ & + & + & + & + & + & + & + & + & + & + & + & + & + & + \\
\hline nagB & ESA_02661 & $\begin{array}{l}\text { Glucosamine-6- } \\
\text { phosphate deaminase }\end{array}$ & + & + & + & + & + & + & + & + & + & + & + & + & + & + \\
\hline neuc & ESA_03772 & $\begin{array}{l}\text { UDP-N- } \\
\text { acetylglucosamine 2- } \\
\text { epimerase }\end{array}$ & + & + & + & + & + & + & + & + & + & + & + & + & + & + \\
\hline siaPQM & $\begin{array}{c}\text { ESA_04264- } \\
4266\end{array}$ & $\begin{array}{l}\text { tripartite ATP- } \\
\text { independent } \\
\text { periplasmic (TRAP) } \\
\text { transporter }\end{array}$ & + & + & + & + & + & + & + & + & + & + & + & + & + & + \\
\hline
\end{tabular}


have increased sialic acid and $\mathrm{N}$-acetylglucosamine residues whereas adults have increased mannose, glucose, and fucose residues [17,18]. Finally, the brain is the major site of sialic acid display in the form of gangliosides (sialylated glycolipids) and sialic acid may have a role in the structural and functional establishment of synaptic pathways [9]. It is possible that, like Neisseria meningitidis, Streptococcus pneumoniae and Haemophilus influenzae which cause bacterial meningitis in children $<5$ years, that C. sakazakii has a developmental dependence on access to the central nervous system.

Given the common clinical association of C. sakazakii infection of neonates with necrotising enterocolitis and brain damage, an improved understanding of sialic acid utilisation by the organism was warranted. This paper describes the plausible link between the recent evolution of sialic acid metabolism by C. sakazakii and its pathogenicity as well as re-investigates the possible presence of sialidase activity.

\section{Results}

Distribution of sialic acid utilization genes

The distribution of sialic acid metabolism genes in Cronobacter and other related Enterobacteriaceae is shown in Table 1 . Essentially nanAKRTC and $y h c H$ were only found in the genomes of C. sakazakii and none of the other Cronobacter species. These genes were also found in E. coli K1 strain CE10 and Cit. koseri, and were not in the related Enterobacteriaceae members Pantoea spp. or E. cloacae. NeuC encoding for UDP-N-acetylglu cosamine 2-epimerase was found in all strains. The genes neuA, nеив, nеuD, nеuO and neuS were absent from all the Cronobacter spp. genomes. NeuA and neuB were only found in E. coli K1 strain CE10.

\section{$\% \mathrm{GC}$ content of nan genes}

As a possible indicator of horizontal gene transfer events the \%GC content of the sialic acid utilization genes was calculated and compared with the rest of the Crono bacter genome. The nanA, nanR and nanT genes of the

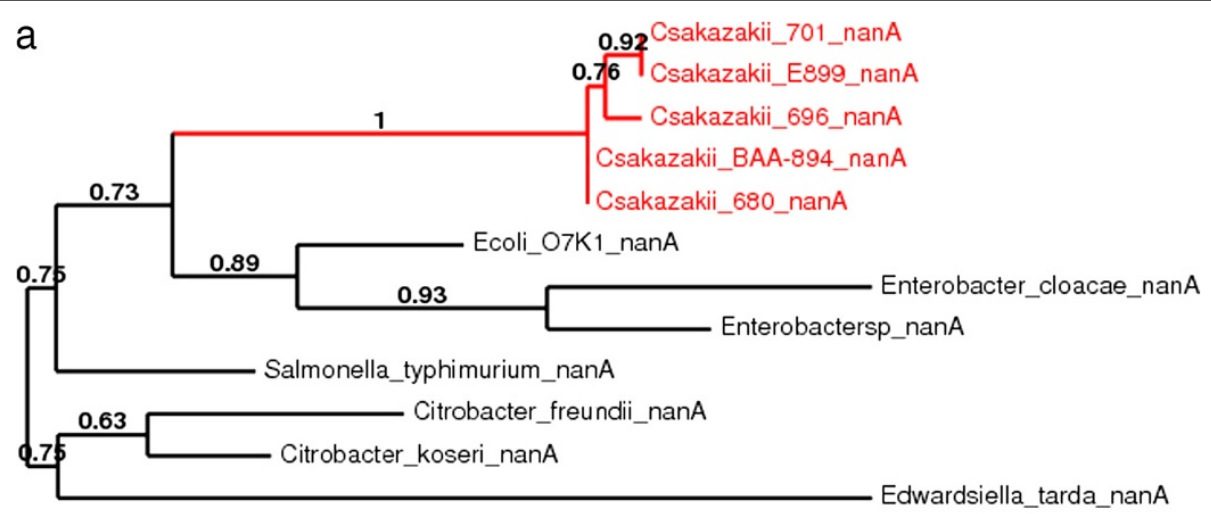

0.08

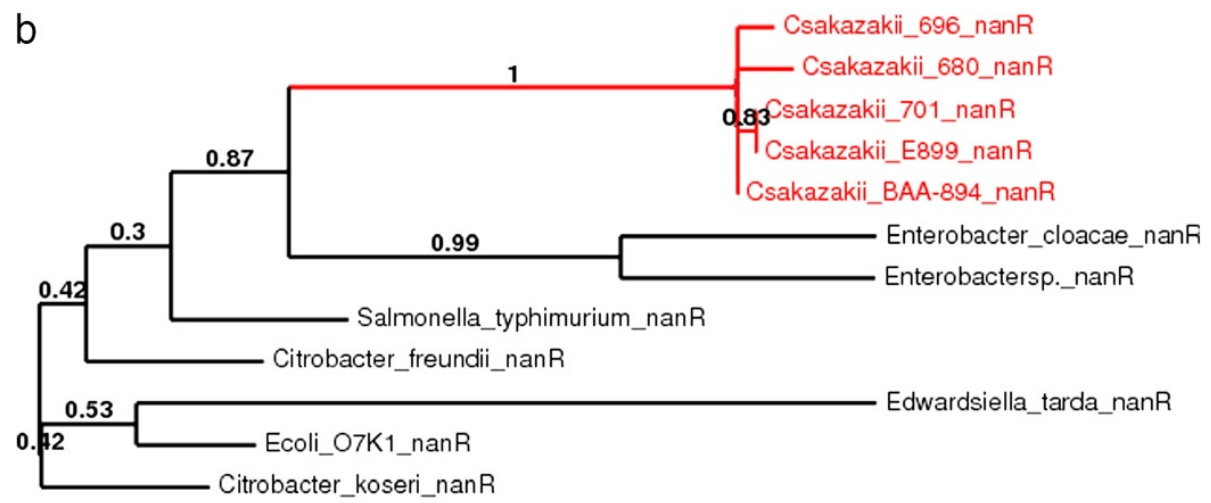

0.08

Figure 1 Maximum-likelihood tree of (a) the NanA protein sequences (292 aa) and (b) NanR protein sequences (260 aa) of C. sakazakii and related Enterobacteriaceae members, constructed using PhyML, with 1000 bootstrap replicates. 
five C. sakazakii genomes showed average GC\% values of $57.2 \%, 56.32 \%$ and $57.14 \%$ respectively which is similar to the average $56 \%$ GC content of the Cronobacter genomes. In contrast, nanE and nanK showed slightly higher values of $63 \%$ and $62.2 \%$ respectively, whereas nanC had a much lower\%GC content value of $47.4 \%$. This was also found for the nanC gene in the closely related organisms Cit. koseri (48\%), E. cloacae (51\%), Enterobacter sp. (44.54\%), and E. coli $\mathrm{K} 1$ (43\%). The genomes of these organisms are between $55-58 \%$ GC. The $n a g A$ and $n a g B$ genes showed GC\% values of $56.3 \%$ and $54 \%$ respectively.

\section{Phylogenetic analysis}

The predicted amino acid sequences of the proteins encoded by these nan cluster genes were analysed and their phylogenetic relationships with closely related Gram-negative bacteria is shown in Figures 1, 2, 3 and 4. Each predicted protein sequence from C. sakazakii formed an independent cluster, with the other Enterobac teriaceae members clustering on the neighbouring branches. In the NanA and NanR (Figure 1) phylogenetic trees, the C. sakazakii cluster appeared to evolve on the same branch as E. cloacae and Enterobacter spp., with the others forming a separate clade. In comparison, the NanK and NanT (Figure 2) C. sakazakii clusters appear to have greater phylogenetic distance from the other enteric members, with a clear split of the population into two clades, one of them being that of the $C$. sakazakii cluster. The nanE gene is found across the Cronobacter genus, and the phylogenetic analysis of the NanE protein sequences (Figure 3) revealed the Cronobacter cluster to have a common and closely related evolutionary clade with E. cloacae, E. hormaechei, Enterobacter spp., Cit. freundii and Pantoea agglome rans. The NanC protein in the C. sakazakii genomes could be located with $>50 \%$ homology only in the genomes of E. cloacae, E. hormaechei, Cit. koseri and E. coli $\mathrm{K} 1$. Of these, the E. coli $\mathrm{K} 1 \mathrm{NanC}$ appeared to be very distantly related to the rest of the population

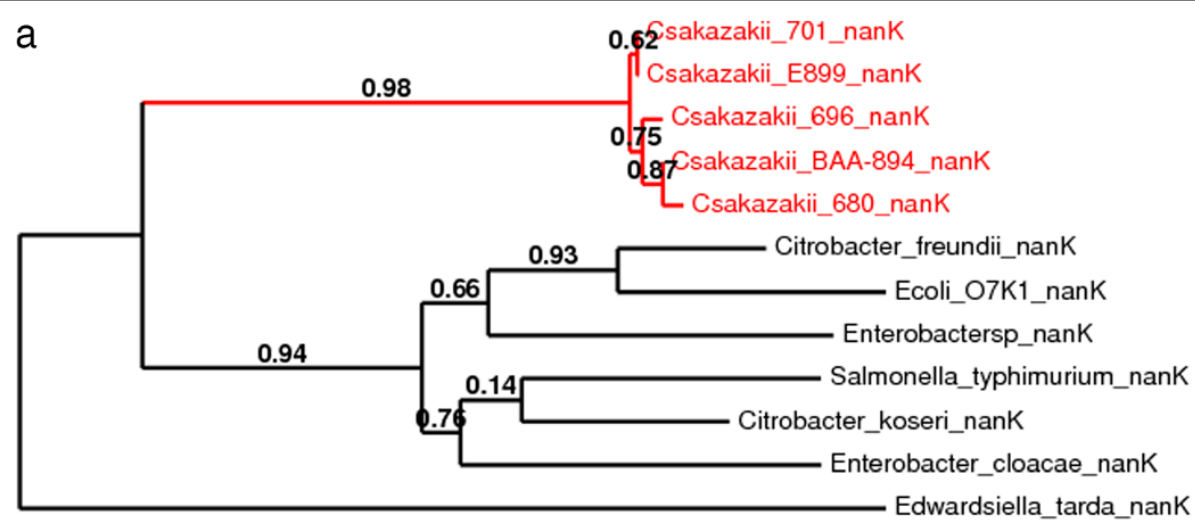

0.2

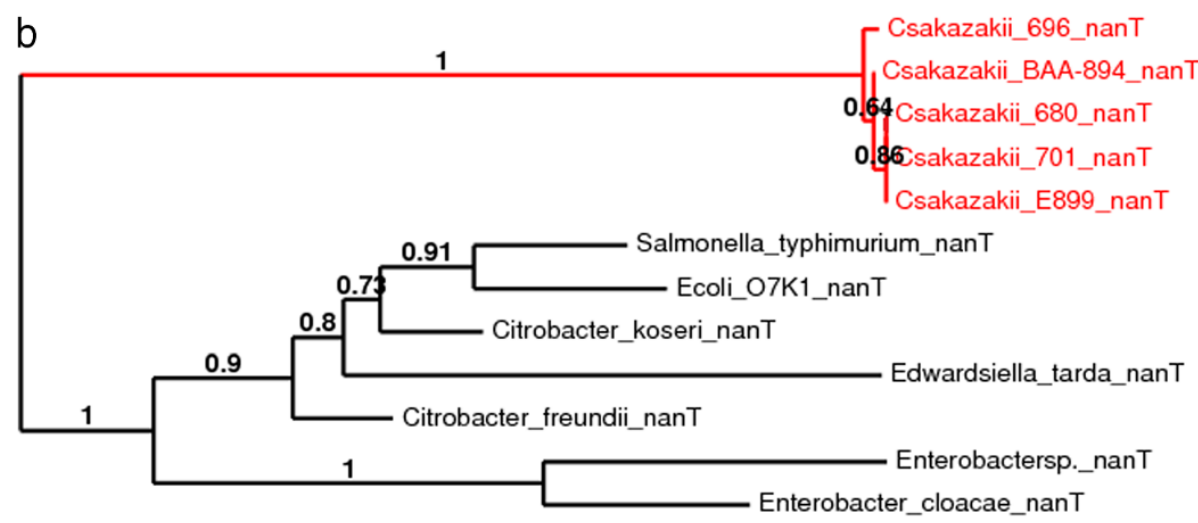

0.07

Figure 2 Maximum-likelihood tree of (a) the NanK protein sequences (291 aa) and (b) NanT protein sequences (496 aa) of C. sakazakii and related Enterobacteriaceae members, constructed using PhyML, with 1000 bootstrap replicates. 


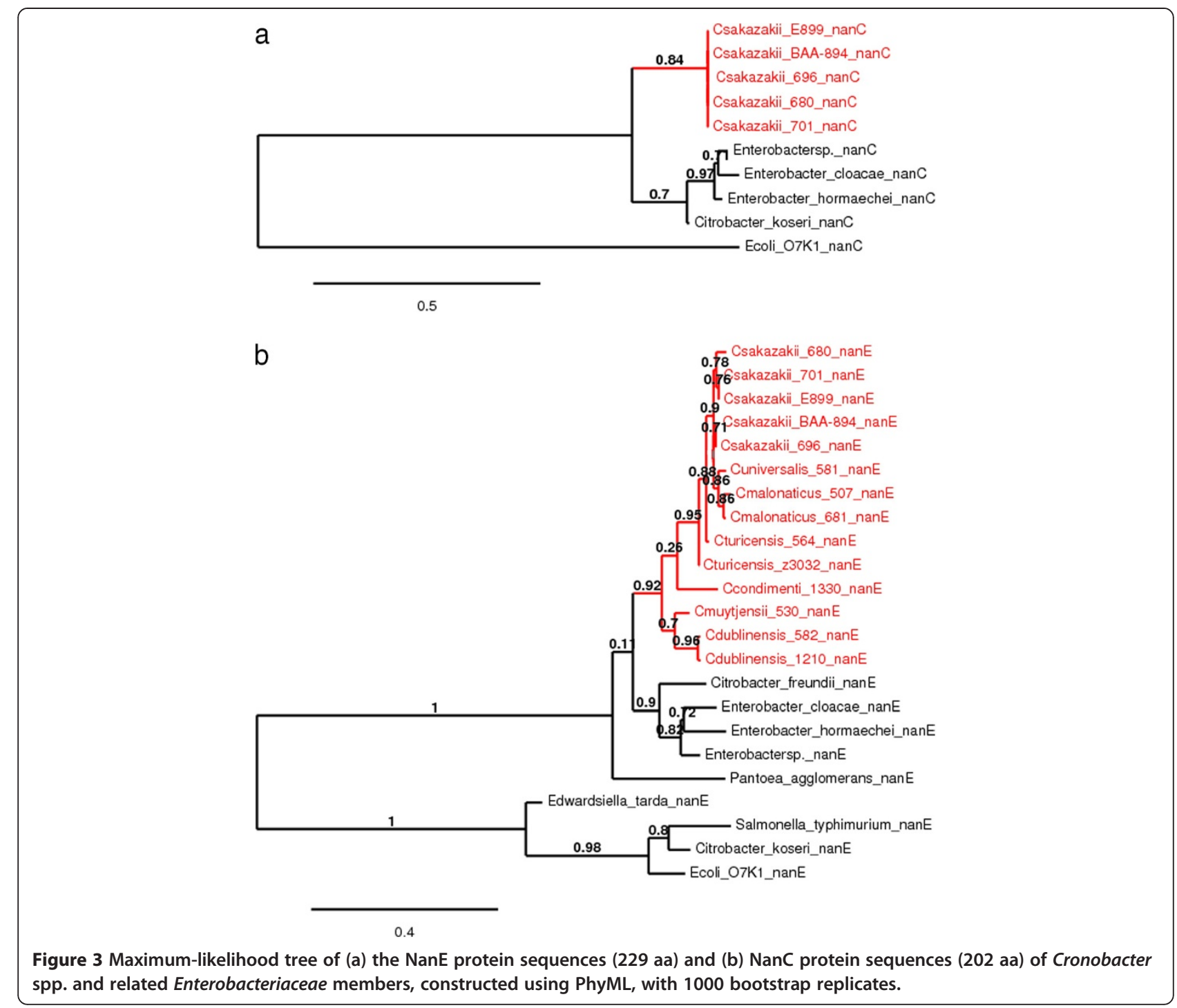

studied (Figure 3). Both the nagA and nagB genes were found in the genomes of all the Cronobacter species (Figure 4). Phylogenetic analysis of these proteins showed the Cronobacter spp. sequences formed a distinct clade, with other Enterobacteriaceae members forming a neighbouring clade, both with a common evolutionary lineage. The newly identified species $C$. condimenti always branched within the Cronobacter genus cluster (Figures 3 and 4). Phylogenetic analysis of Cit. koseri and Cit. freundii nan gene sequences revealed different patterns in their branching, which indicates possible independent evolutionary paths for the nan genes within the Citrobacter genus.

\section{Growth of C. sakazakii on sialic acid and GM1}

The sialic acid utilization pathway was confirmed in 6 strains of C. sakazakii by their growth in minimal medium (M9) with sialic acid as the sole carbon source; Figure 5.
There was no growth by any strains $(\mathrm{n}=8)$ of the other six Cronobacter species. Cit. koseri, Cit. freundii and Ed. tarda which also have predicted nan genes grew in M9 supplemented with sialic acid. Growth was also observed for C. sakazakii strains, and Cit. koseri in minimal medium supplemented with the monosialoganglioside GM1 indicating possible sialidase activity; Figure 5 See Additional file 1: Table S1. No growth was observed for any organisms in M9 in the absence of a carbon source. Since the sialidase gene $n a n H$ had not previously been described for either of C. sakazakii or Cit. koseri, a bioinformatics re-analysis of their genomes was undertaken.

\section{Candidate sialidase genes in C. sakazakii}

As in previous studies, BLAST searches of the Crono bacter genomes for homology to the well characterised sialidase gene (nanH) from Haemophilus influenzae and Vibrio cholerae did not result in any matches. However 


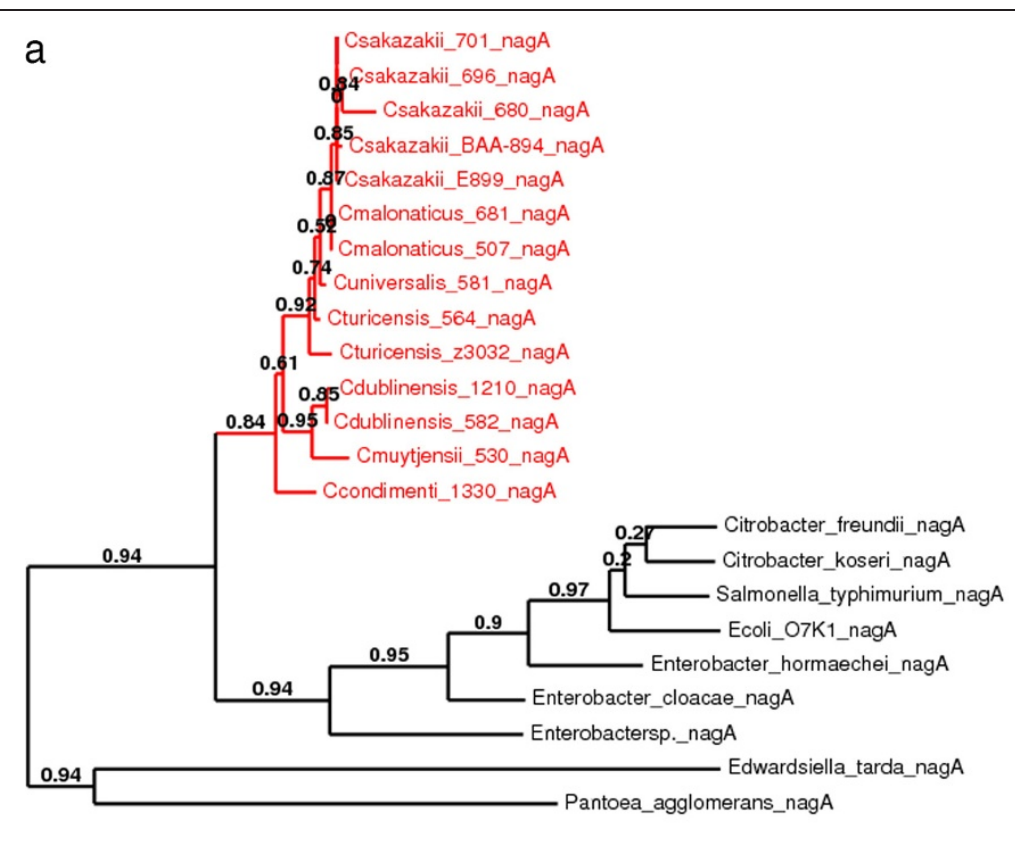

0.2

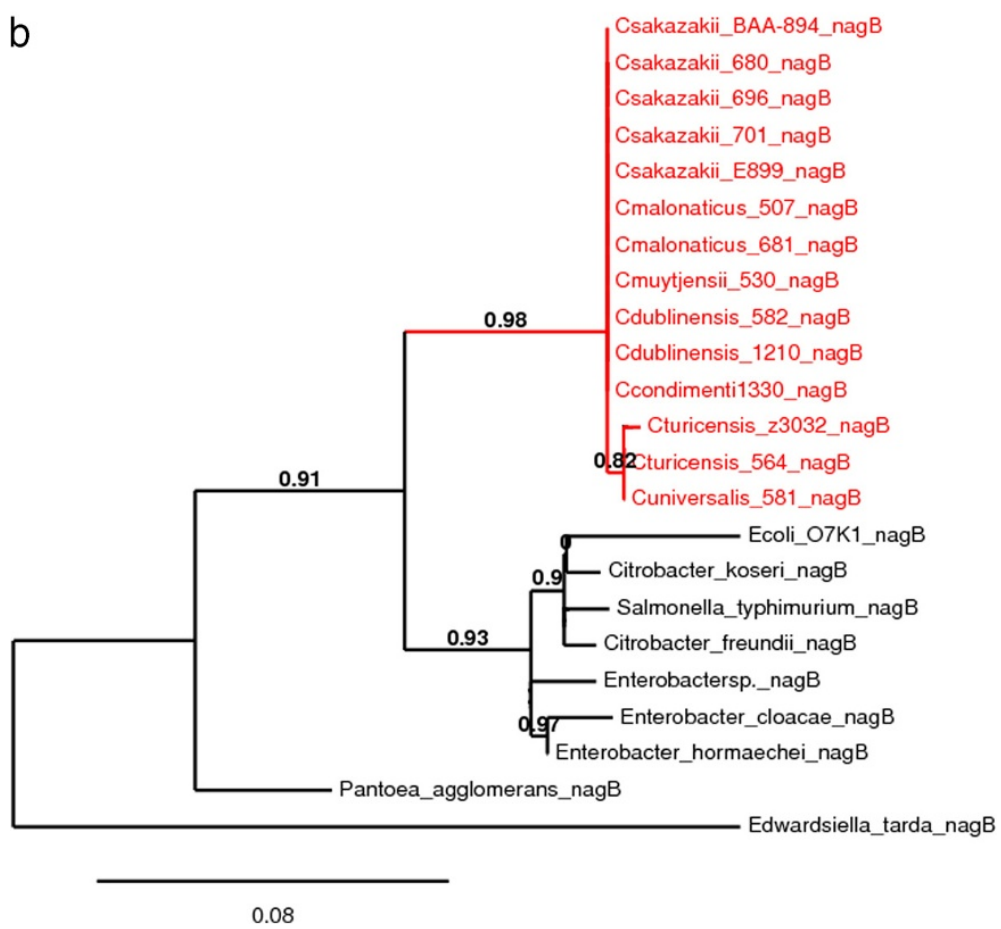

Figure 4 Maximum-likelihood tree of (a) the NagA protein sequences (382 aa) and (b) NagB protein sequences (266 aa) of Cronobacter spp. and related Enterobacteriaceae members, constructed using PhyML, with 1000 bootstrap replicates.

given the low homology $(<30 \%)$ between sialidases the Cronobacter genomes were further investigated for the characteristic sialidase RIP and Asp box motifs [13]. The Asp box motif is composed of repeated Ser/Thr-x-Asp$x$-Gly- $x$-Thr-Trp/Phe regions, where $x$ represents any amino acid. No candidate genes were found encoding the RIF region and Asp box motifs in the C. sakazakii genome.

\section{Discussion}

C. sakazakii is estimated to have branched from the rest of the Cronobacter members approximately 15-23 MYA 

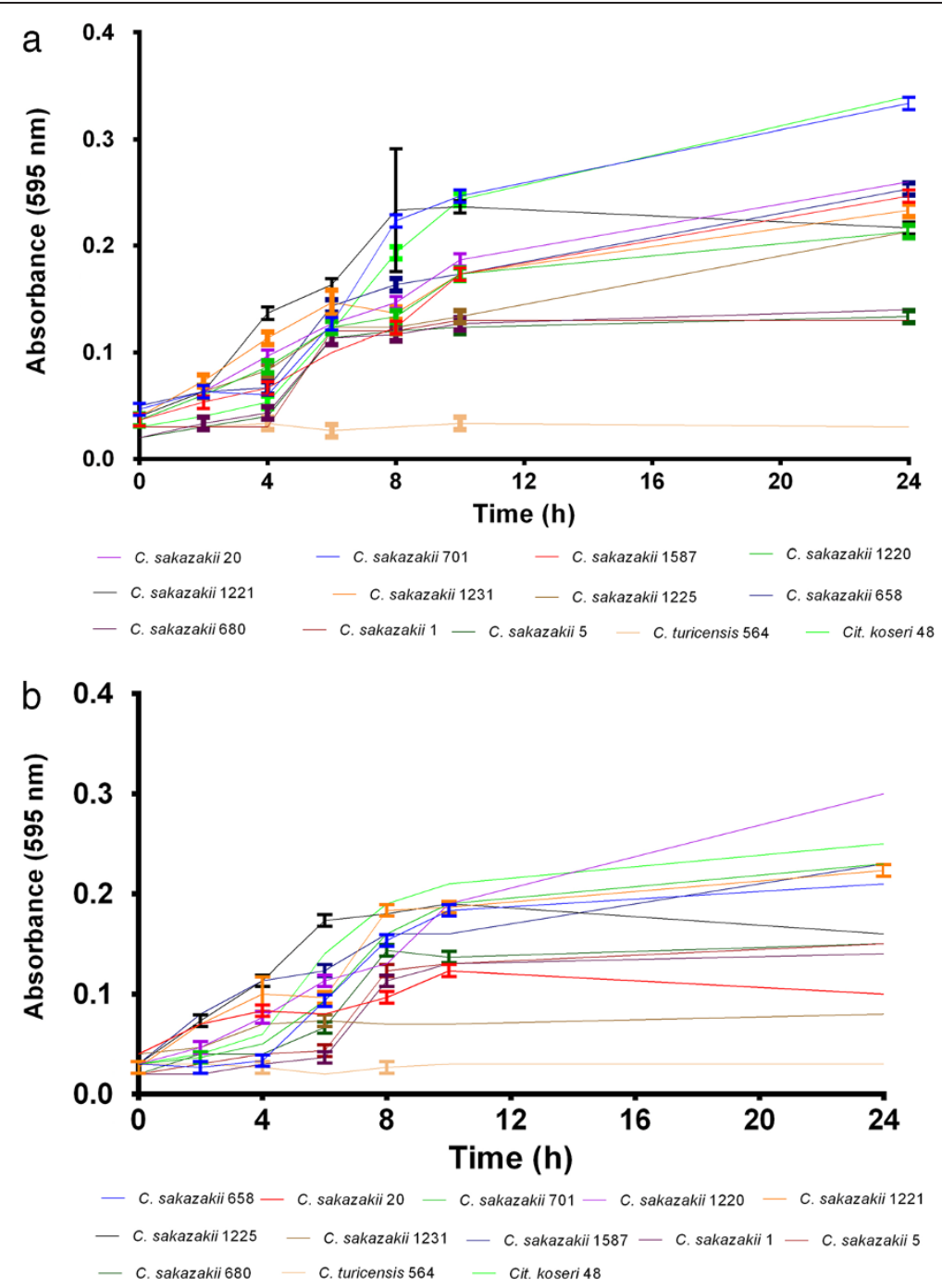

Figure 5 Growth of Cronobacter sakazakii in M9 minimal medium supplemented with (a) sialic acid, and (b) GM1 ganglioside as sole carbon source.

[6], and is the Cronobacter species most frequently isolated from clinical infections of neonates. The absence of the core sialic acid utilization genes in the Cronobacter genus except for C. sakazakii, suggests their acquisition was by horizontal gene transfer instead of gene loss by the rest of the genus. The metabolism of sialic acid could have a role in the organisms' virulence. The evolution of $C$. sakazakii post-dates key eras in the evolution of sialic acid biosynthetic pathway which occurred near the divergence of Coelomata (protostomes and deuterostomes ca.500 MYA), and milk producing mammals (Jurassic Era ca.200145 MYA). Therefore the presence of sialic acid is a relatively recent evolution, and high levels are accessible to bacteria in milk, the intestines, and the brain.

The C. sakazakii genomes possess the genes required for the uptake and catabolism of sialic acid; Table 1. Although the nanAKTER genes are usually clustered together, in C. sakazakii nanE is located at a different region (ESA_00529) from the others (ESA_03610-12). This split has also been reported for Ed. tarda and Cit. freundii [14]. This possibly reflects a separate evolutionary lineage for the gene. All the C. sakazakii genomes encode for the NanC outer membrane porin and NanT inner membrane transporter protein. Hence, all $C$. sakazakii strains can potentially transport the exogenous sialic acid into the cytoplasm of their cells. Interestingly, all the Cronobacter species genomes also exhibited the presence of the genes (siaPQM) encoding for the TRAP transporter; Table 1. However laboratory growth studies confirmed that C. sakazakii was the only Cronobacter species which could grow on sialic acid as a carbon source and indicates that NanT is the active transporter in the process; Figure 5a. The acquisition of genes encoding for the utilization of exogenous sialic acid may have a 
major role in C. sakazakii colonisation of the human intestinal tract (via mucins) and the use of sialic acid in breast milk or infant formula as a nutrient source $[9,18]$.

The demonstration of $C$. sakazakii growth on the ganglioside GM1 (Figure 5b) indicated the possible possession of a sialidase enzyme, which despite previous sequence searches for nanH (the gene encoding for sialidase) had not been reported. Since the homology between sialidases is very low $(<30 \%)$ [13], a more detailed search for the sialidase RIP and Asp-box motifs was undertaken. However no candidate genes were found with both RIP and Asp-box motifs. Nevertheless the growth on GM1 indicated a ganglioside degradative ability by C. sakazakii. GM1 is composed of galactose, $\mathrm{N}$-acetyl-galactose, glucose as well as sialic acid units linked by $\beta 1-3$ and $\beta 1-4$ linkages, and these are joined to a steroid. Therefore it is proposed that GM1 is degraded to metabolisable sugar units by the activity of various $\beta$-galactosidases (ESA_01827, ESA_02977 \& ESA_03417), $\beta$-acetyl-hexosaminidases (ESA_02237 and ESA_02655), esterases (ESA_00377 \& ESA_00776) and lipases (ESA_02127 \& ESA_02202), and therefore able to grow on this substrate.

The \%GC content of the nanC gene was found to be $47 \%$, considerably less than the $56 \%$ GC content of the overall Cronobacter genome. Slight aberrations were also seen in the \%GC content values of the nanK and nanE genes, an observation previously noted in an evolutionary study of the nan clusters of other Enterobacteriaceae members such as Salmonella enterica, E. coli and Yersinia spp. [11]. These observations suggest a strong possibility of horizontal transfer events having influenced separate acquisitions for the nanAKT cluster in $C$. sakazakii, as well as the $n a n C$ and $n a n E$ clusters in the whole genus. Hence, it is possible that the nan clusters could have evolved in a mosaic pattern in this bacterial genus. A phylogenetic analysis was also conducted using the protein sequences encoded by the sialic acid utilization genes in C. sakazakii (Figures 1, 2, 3 and 4). This revealed that the nanATKR genes have evolved as a lineage in C. sakazakii, and independent of other closely related pathogens of the Enterobacteriaceae family. No close homology (50\% cut-off) was found between the NanC protein sequences and those from most other members of the Enterobacteriaceae. This indicates the acquisition of the associated gene in C. sakazakii was from a more distantly related organism, currently unidentified.

It is also notable that the nanATK cluster in $C$. sakazakii is located adjacent to a stringent starvation gene homologue (sspA, ESA_03615) and therefore expression of this gene cluster could be responsive to environmental nutrient levels; Table 1 . The presence of other related genes such as nagA and nagB, leading to the synthesis of fructose-6-phosphate, support the role of sialic acid as a carbon source in C. sakazakii. Whereas the absence of genes such as neuS, neuO and neuD in the genomes of the Cronobacter genus indicates that the organism does not decorate its cell surface with sialic acid, unlike E. coli $\mathrm{K} 1$ and $N$. meningitidis [10,14].

\section{Conclusions}

Three rich mammalian sources of sialic acid for pathogenic or commensal bacteria are in the gastrointestinal tract, the brain and in human milk. These three locations of sialic acid correlate with the clinical presentations of C. sakazakii infections with respect to necrotizing enterocolitis and the intensive brain damage during neonatal meningitis. This is especially pertinent since most of the neonatal infections in $C$. sakazakii have been reported at a very early stage of growth with half in the first week and three quarters during the first month of age $[1,2,4]$. Apart from these mammalian sources of sialic acid, another feature with the organism is that sialic acid is often added to infant formula, a

Table 2 Bacterial strains used for laboratory studies of growth on sialic acid and GM1 as sole carbon source

\begin{tabular}{|c|c|c|c|}
\hline Organism & Strain & Sequence type $^{a}$ & Source \\
\hline C. sakazakii & $1^{\mathrm{b}}$ & ST8 & Throat \\
\hline C. sakazakii & 5 & ST8 & Blood \\
\hline C. sakazakii & 658 & ST1 & Non-infant formula \\
\hline C. sakazakii & 680 & ST8 & Cerebral spinal fluid \\
\hline C. sakazakii & 696 & ST12 & Faeces \\
\hline C. sakazakii & 701 & ST4 & Peritoneal fluid \\
\hline C. sakazakii & 1220 & ST4 & Cerebral spinal fluid \\
\hline C. sakazakii & 1221 & ST4 & Cerebral spinal fluid \\
\hline C. sakazakii & 1225 & ST4 & Blood \\
\hline C. sakazakii & 1231 & ST4 & Faeces \\
\hline C. sakazakii & 1587 & ST4 & Cerebral spinal fluid \\
\hline C. malonaticus & 507 & ST11 & Faeces \\
\hline C. malonaticus & $681^{c}$ & ST7 & Breast abscess \\
\hline C. universalis & 581 & ST54 & Water \\
\hline C. turicensis & 564 & ST5 & Blood \\
\hline C. turicensis & $1211^{d}$ & ST19 & Blood \\
\hline C. muytjensii & 530 & ST49 & Powdered infant formula \\
\hline C. dublinensis & 582 & ST36 & Unknown \\
\hline C. condimenti & $1330^{\mathrm{e}}$ & ST40 & Spiced sausages \\
\hline Ed. tarda & 1926 & - & Unknown \\
\hline Cit. freundii & 1927 & - & Unknown \\
\hline Cit. koseri & 48 & - & Clinical \\
\hline
\end{tabular}

a. Sequence type as according to the Cronobacter genus multilocus sequence typing scheme database; http://www.pubMLST.org/cronobacter.

b. C. sakazakii species type strain (ATCC $29544^{\top}$ ).

c. C. malonaticus species type strain (LMG $23826^{\top}$ ).

d. C. turicensis species type strain (LMG 28327 $)$.

e. C. condimenti species type strain. 
recognised source of neonatal Cronobacter infections $[4,6]$. The unique utilization of sialic acid by $C$. sakazakii indicates a plausible adaptation of the organism to host. The source of the nan genes is not fully understood, but appears to be by multiple horizontal gene transfers, the sources of which are uncertain to date.

\section{Methods}

\section{Strains and culture conditions}

Nineteen Cronobacter strains were selected which represented the seven recognized species, and included those from reported clinical cases and species type strains (Table 2). All Cronobacter strains were stored at $-80^{\circ} \mathrm{C}$ in Nutrient Broth (Oxoid, UK) with 10\% glycerol, subcultured on Trypticase Soy Agar (Oxoid ThermoFisher, UK) and checked for purity. Cit. koseri strain 48, Cit. freundii 1927, Ed. tarda 1926 were included for comparative purposes.

\section{Sialic acid utilization and sialidase degradation determination}

The method of Almagro-Moreno and Boyd [11] was followed with two modifications. The bacterial cultures were grown overnight in Brain Heart Infusion broth (Oxoid ThermoFisher, UK), and the cell suspension was washed three times before the assay to remove the carryover of nutrients. Growth in M9 minimal media with sialic acid (1 $\mathrm{mg} / \mathrm{ml}$, Sigma Aldrich), or GM1 monosialoganglioside (1 $\mathrm{mg} / \mathrm{ml}$, Sigma Aldrich) as the carbon source was measured by absorbance increase at $595 \mathrm{~nm}$. Inoculation of M9 without a carbon source was used as a negative control, and glucose was added $(1 \mathrm{mg} / \mathrm{ml})$ to the minimal medium as a positive control.

\section{Phylogenetic analysis of sialic acid utilization genes}

The amino acid sequences of the genes nanK, nanT, nanE, nanA, nanC, nagA and nagB from the fourteen Cronobacter spp. genomes (CALG01000001-C201, CAL F01000001-569, CALE01000001-768, AFMO00000000, NC_009778-80, CALD01000001-249, CALC01000001171, CALB01000001-114, NC_013282-85, CALA01000 001-427, CAKZ01000001-221, CAKY01000001-365, CA KX01000001-231, CAKW01000001-155) were obtained from RAST prokaryotic genome annotation server. The gene sequences from the genome of $C$. sakazakii BAA-894 was used to perform tblastx searches to obtain the corresponding sequences of closely related organisms showing $>50 \%$ amino acid identity. The corresponding protein sequences were downloaded from the genomes of $E$. coli O7:K1 strain CE10 (Accession No. NC_017646); E. cloacae subsp. cloacae ATCC 13047 (Accession No. NC_014121); E. hormaechei ATCC 49162 (Accession No. AFHR00000000); Enterobacter spp. strain 683 (Accession No. CP000653); Cit. freundii strain 4_7_47CFAA (Accession No. ADLG00000000); Cit. koseri ATCC BAA-895 (Accession No. NC_009792); Ed. tarda strain EIB202 (Accession No. NC_013508); Salmonella enterica subsp. enterica serovar Typhimu rium strain LT2 (Accession No. NC_003197); Pantoea agglomerans strain IG1 (Accession No. BAEF00000000). The sequences of each gene were aligned using ClustalW [19] and phylogenetic analysis was performed using the Maximum-Likelihood algorithm in PhyML $[20,21]$. Stability of the relationships was assessed by the bootstrap method (1000 replicates) and the trees were viewed and annotated using TreeDyn at http://www. phylogeny.fr [22,23].

\section{Additional file}

Additional file 1: Table S1. Growth of Cronobacter sakazakii in M9 minimal medium supplemented with (a) sialic acid, and (b) GM1 ganglioside as sole carbon source.

\section{Competing interests}

The authors declare that they have no competing interests.

\section{Authors' contributions}

SJ and NM carried out the genome sequence analysis. SH undertook the laboratory studies. SH and SJF designed the laboratory studies. SJF conceived the study. SJ and SJF drafted the manuscript. All authors read and approved the final manuscript.

\section{Acknowledgements}

The authors thank Nottingham Trent University for their financial support of SJ and NM, and the Amm-AlQura University for their financial support of SH. The authors also thank Prof Nadia Chuzhanova for her assistance in bioinformatics analysis.

Received: 13 February 2013 Accepted: 16 May 2013 Published: 24 May 2013

\section{References}

1. Centers for Disease Control and Prevention: CDC Update: investigation of Cronobacter infections among infants in the United States. 2012. http://www. cdc.gov/cronobacter/investigation.html.

2. Hariri S, Joseph S, Forsythe SJ: Predominance of Cronobacter sakazakii ST4 clonal complex strains in Cronobacter neonatal meningitis infections in US 2011. Emerg Infect Dis 2013, 19:175-177.

3. Townsend SM, Hurrell E, Gonzalez-Gomez I, Lowe L, Frye JG, Forsythe S, Badger J: Enterobacter sakazakii invades brain capillary endothelial cells, persists in human macrophages influencing cytokine secretion and induces severe brain pathology in the neonatal rat. Microbiol 2007, 153:3538-3547.

4. Kucerova E, Joseph S, Forsythe S: Cronobacter: diversity and ubiquity. Qual Assur Safety Foods Crops 2011, 3:104-122.

5. Joseph S, Cetinkaya E, Drahovske H, Levican A, Figueras MJ, Forsythe SJ: Cronobacter condimenti sp. nov., isolated from spiced meat and Cronobacter universalis sp. nov., a novel species designation for Cronobacter sp. genomospecies 1, recovered from a leg infection, water, and food ingredients. Intl J Syst Evol Microbiol 2012, 62:1277-83.

6. Joseph S, Sonbol H, Hariri S, Desai P, McClelland M, Forsythe SJ: Diversity of the Cronobacter genus as revealed by multi locus sequence typing. $J$ Clin Microbiol 2012, 50:3031-3039.

7. Kucerova E, Clifton SW, Xia X-Q, Long F, Porwollik S, Fulton L, Fronick C, Minx P, Kyung K, Warren W, Fulton R, Feng D, Wollam A, Shah N, Bhonagiri V, Nash WE, Hallsworth-Pepin K, Wilson RK, McClelland M, Forsythe SJ: Genome sequence of Cronobacter sakazakii BAA-894 and comparative genomic 
hybridization analysis with other Cronobacter species. PLoS One 2010, 5: e9556.

8. Joseph S, Desai P, Li Y, Cummings CA, Shih R, Degoricija L, Rico A, Brzoska P, Hamby SE, Masood N, Hariri S, Sonbol H, Chuzhanova N, McClelland M, Furtado MR, Forsythe SJ: Comparative analysis of genome sequences covering the seven Cronobacter species. PLoS One 2012, 7:e49455.

9. Wang B: Sialic acid is an essential nutrient for brain development and cognition. Ann Rev Nutr 2009, 29:177-222

10. Severi E, Hood DW, Thomas GH: Sialic acid utilization by bacterial pathogens. Microbiol 2007, 153:2817-2822.

11. Almagro Moreno S, Fidelma Boyd E: Insights into the evolution of sialic acid catabolism among bacteria. BMC Evol Biol 2009, 9:118.

12. Roggentin P, Schauer R, Hoyer LL, Vimr ER: The sialidase superfamily and its spread by horizontal gene transfer. Mol Microbiol 1993, 9:915-921.

13. Kim S, Oh DB, Kang HA, Kwon O: Features and applications of bacterial sialidases. Appl Microbiol Biotechnol 2011, 91:1-15.

14. Vimr E: Unified theory of bacterial sialometabolism: how and why bacteria metabolize host sialic acids. ISRN Microbiology Volume 2013 (2013), Article ID 816713. doi:http://dxorg/10.1155/2013/816713.

15. Caubilla-Barron J, Hurrell E, Townsend S, Cheetham P, Loc-Carrillo C, Fayet O, Prere M-F, Forsythe SJ: Genotypic and phenotypic analysis of Enterobacter sakazakii strains from an outbreak resulting in fatalities in a neonatal intensive care unit in France. J Clin Microbiol 2007, 45:3979-3985.

16. Sun Y, Arbatsky NP, Wang M, Shashkov AS, Liu B, Wang L, Knirel YA: Structure and genetics of the $\mathrm{O}$-antigen of Cronobacter turicensis G3882 from a new serotype, $\mathrm{C}$. turicensis $\mathrm{O} 2$, and identification of a serotype-specific gene. FEMS Immun Med Microbiol 2012, 66:323-333.

17. Sprenger N, Duncan PI: Sialic acid utilization. Adv Nutr 2012, 3:392S-397S.

18. Lewis AL, Lewis WG: Host sialoglycans and bacterial sialidases: a mucosal perspective. Cell Microbiol 2012, 14:1174-1182.

19. Thompson JD, Higgins DG, Gibson TJ: CLUSTAL W: improving the sensitivity of progressive multiple sequence alignment through sequence weighting, position-specific gap penalties and weight matrix choice. Nucleic Acids Res 1994, 22:4673-80

20. Felsenstein J: Evolutionary trees from DNA sequences: a maximum likelihood approach. J Mol Evol 1981, 17:368-376.

21. Guindon S, Gascuel O: A simple, fast and accurate algorithm to estimate large phylogenies by maximum likelihood. System Biol 2003, 52:696-704.

22. Chevenet F, Brun C, Banuls AL, Jacq B, Christen R: TreeDyn: towards dynamic graphics and annotations for analyses of trees. BMC Bioinform 2006, 7:439.

23. Dereeper A, Guignon V, Blanc G, Audic S, Buffet S, Chevenet F, Dufayard JF, Guindon S, Lefort V, Lescot M, Claverie JM, Gascuel O: Phylogeny.fr: robust phylogenetic analysis for the non-specialist. Nucleic Acids Res 2008, 36:W465-W469.

doi:10.1186/2042-5783-3-3

Cite this article as: Joseph et al: Sialic acid utilization by Cronobacter sakazakii. Microbial Informatics and Experimentation 2013 3:3.

\section{Submit your next manuscript to BioMed Central and take full advantage of:}

- Convenient online submission

- Thorough peer review

- No space constraints or color figure charges

- Immediate publication on acceptance

- Inclusion in PubMed, CAS, Scopus and Google Scholar

- Research which is freely available for redistribution

Submit your manuscript at www.biomedcentral.com/submit
Biomed Central 